\title{
Diffuse gamma-ray emission modeling and the Galactic center $\mathrm{GeV}$ excess
}

\section{Dmitry Malyshev*t}

ECAP, Erwin-Rommel-Str. 1, D-91058, Erlangen, Germany

E-mail: dmitry.malyshevafau.de

The Galactic center is one of the most important regions to search for a gamma-ray signal from a possible annihilation of dark matter due to its proximity and high dark matter density. In the past years, several groups have reported an excess of gamma rays in the Fermi Large Area Telescope data with an approximately spherical morphology around the Galactic center. In this contribution, we will overview the current observational status of the excess and the difficulties in the gammaray analysis near the Galactic center.

7th Fermi Symposium 2017

15-20 October 2017

Garmisch-Partenkirchen, Germany

* Speaker.

${ }^{\dagger}$ On behalf of the Fermi-LAT collaboration. 


\section{Introduction}

Galactic center (GC) is one of the most important regions to search for a possible gamma-ray signal from annihilation of dark matter (DM) particles. It is also one of the most complex regions in the sky with many sources of gamma rays contributing along the line of sight, e.g., interactions of cosmic rays (CR) with gas and interstellar radiation fields (ISRF) and point sources (PS). Soon after the Fermi Large Area Telescope (LAT) data became available, there were hints of an excess of gamma rays with a spherical morphology near the GC with a peak in spectral energy distribution around a few $\mathrm{GeV}[1,2,3]$. Later this excess was confirmed by many groups $[4,5,6,7,8,9,10,11]$. Although the excess of gamma rays is consistent with annihilation of DM, one has to investigate carefully the uncertainties in gamma-ray emission near the GC as well as to look for other possible explanations of the excess, such as a population of faint point sources. In this contribution, we will review uncertainties related to analysis of diffuse components as well as resolved point sources near the GC. We will consider interactions of cosmic rays with gas, inverse Compton (IC) component, additional sources of CR near the GC, a model of the Fermi bubbles [12]. We will also discuss uncertainties in the determination of the excess morphology.

\section{Challenges of diffuse gamma-ray analysis}

The largest component of gamma-ray emission along the Galactic plane (GP) is due to interactions of CR with gas. There are several sources of uncertainty related to this component. First of all, the distribution of CR sources and propagation parameters are known only approximately. Usually the calculations are performed with isotropic diffusion of CR [13, 14], but the diffusion may be unisotropic due to preferential direction of the magnetic field along the GP. There can also be convection perpendicular to the plane, especially near the GC, that can transport CR away from the GP. The standard tracers of CR sources, such as supernova remnants, pulsars, OB stars, have a deficit near the GC [15]. However, observations of young stellar objects and free-free emission suggest that the star formation rate (SFR) near the GC can be 5 - 15\% of the total SFR in the Galaxy $[16,17,18]$. The problems of CR sources and propagation can be mitigated by splitting the interactions of gas and CR in rings and fitting each ring to the data independently. This method relies on a knowledge of the gas distribution in the Galaxy, which is typically obtained by measuring the velocity of the gas. If the rotation of the Galactic plane is known, then the velocity information can be translated to the position. A significant caveat is that towards the GC the gas moves perpendicular to the line of sight. As a result, in the standard analysis, the distribution of gas towards the GC is reconstructed from interpolation of gas at longitudes $|\ell|>10^{\circ}$ [15].

The second most important source of gamma rays along the GP is the inverse Compton emission from interactions of high energy CR electrons and positrons with interstellar radiation fields. Apart from uncertainties in CR sources and propagation, which we have already discussed, for the leptonic component there is also an interplay of propagation and energy losses, while there can be significant uncertainties in the density of ISRF near the GC [19]. The interactions of cosmic rays with gas can be traced by the gas distribution in the Galaxy, which has rather distinctive spacial features. The ISRF distribution is generally rather smooth, e.g., there are no distinctive features 
that can be used to trace the IC emission. As a result, overall in the Galaxy, the IC emission is much harder to model than the interactions of $\mathrm{CR}$ with gas.

The third component that one has to take into account is the resolved PS. Point sources can be usually separated from the diffuse emission due to their distinctive "point-like" spatial profile, which is only smeared by the angular resolution of the instrument. Near the GC, the difficulties in modeling the diffuse emission result in over-subtractions, which reduce the probability of a detection of a PS, and under-subtractions, which can lead to detection of spurious PS. Also, due to large density of PS near the GC, a spacial overlap among the PS is possible.

Fermi bubbles (FB) are even harder to model near the GC than the cosmic ray interactions and PS. Provided that there are clear counterparts for the FB at other wavelengths, neither the spectrum nor the morphology of FB near the GC are known. In the following sections we will discuss the uncertainties in the GeV GC excess due to modeling of interactions of CR with gas in the Galaxy, IC emission, CR sources near the GC, resolved PS near the GC, and modeling of FB.

\section{CR sources and propagation in the Galaxy}

In order to set the stage for the discussion we reproduce here in Figure 1 the comparison of GC excess flux within $10^{\circ}$ from the GC relative to the other components of gamma-ray emission in the Sample model of [11]. Around a few $\mathrm{GeV}$, the largest flux in this region is due to $\mathrm{CR}$ interacting with the gas via hadronic collisions (the $\pi^{0}$ component) and bremsstrahlung production of gamma rays by electrons and positrons scattering off gas nuclei. The second largest flux is due to IC scattering. The GC excess is the third largest component, it provides up to 10 - $15 \%$ of the total gamma-ray flux around a few GeV. Below about $1 \mathrm{GeV}$, the GC excess component is subdominant to the flux from

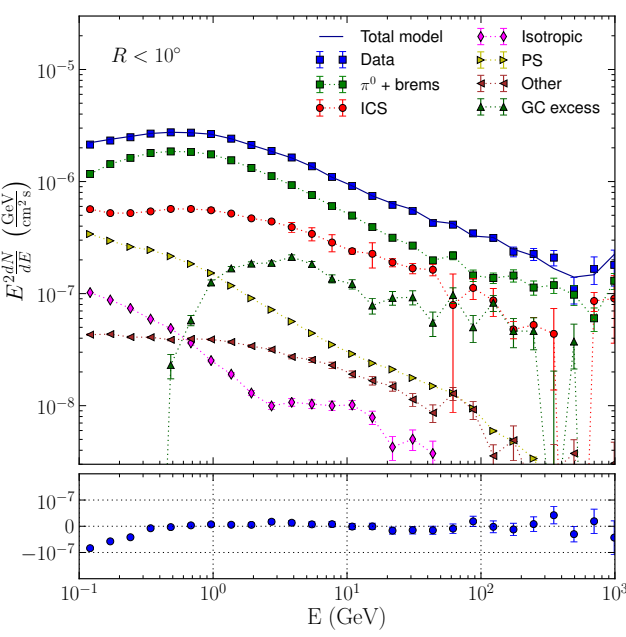

Figure 1: Components of gamma-ray emission within $10^{\circ}$ from the GC in the Sample model of [11]. resolved PS (modeled from the 3FGL catalog). The FB are absent on this plot, because they were modeled for $|b|>10^{\circ}$ in this analysis.

In order to test the influence of modeling the CR source distribution and propagation, one can compare different distributions of sources in the Galaxy and diffusion parameters, such as the propagation halo size. The GC excess flux for different variants of the models is shown in Figure 2. The variations of the GC excess flux are not very large above $1 \mathrm{GeV}$, but can be significant below $1 \mathrm{GeV}$.

An additional source of uncertainty is the distribution of gas along the line of sight towards the GC. In order to test the influence of the gas distribution on the GC excess, an alternative distribution of gas can be derived using starlight extinction to calculate the distribution of dust, then, assuming a homogeneous mixing of dust and gas, a distribution of gas can be estimated [11]. The uncertainty 

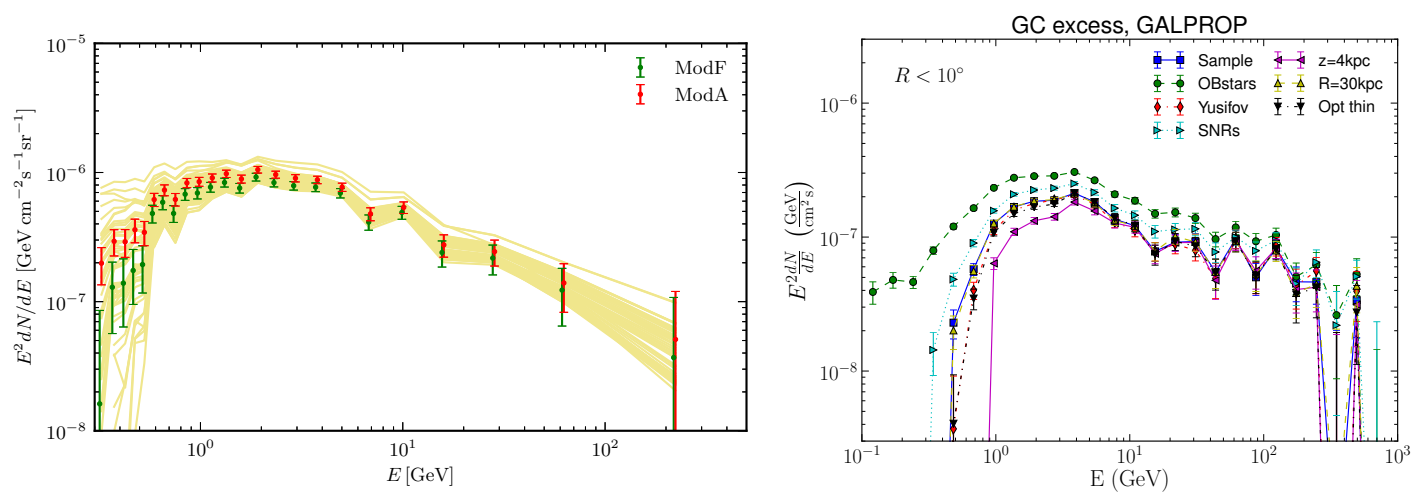

Figure 2: Variations of CR sources distribution and propagation in [8] (left) and [11] (right).

in the gas distribution towards the GC can have a significant effect on the GC excess flux below about $1 \mathrm{GeV}$ (Figure 3 on the left).
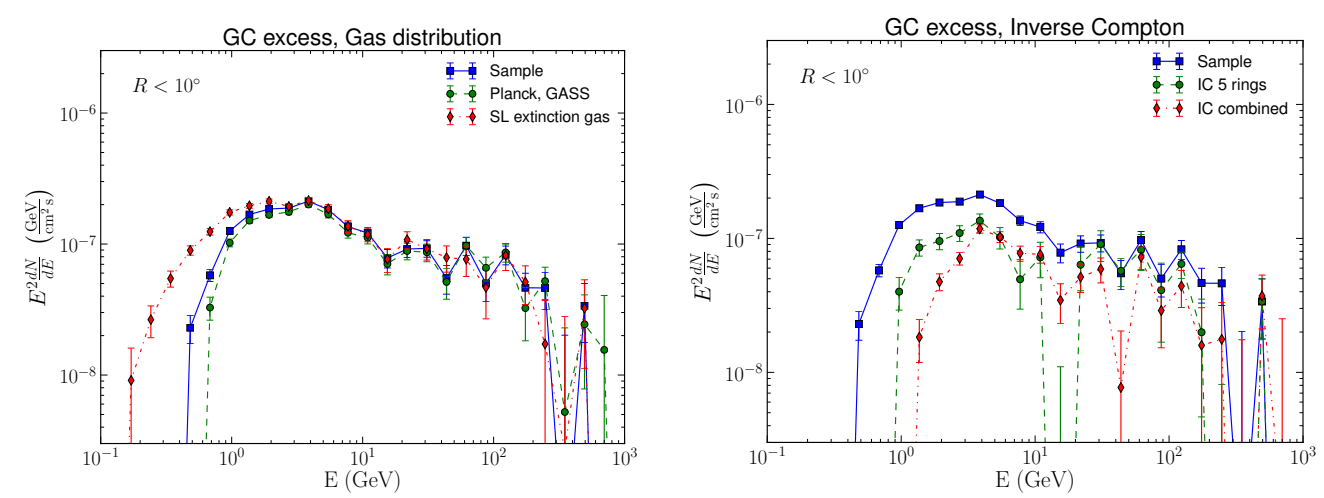

Figure 3: Left: effect of an alternative distribution of gas based on dust estimates from starlight extinction; right: variations of IC emission models [11].

\section{IC component}

The IC emission is relatively difficult to determine due to lack of distinctive spatial features that can be used to trace it. An estimate of the effect of mismodeling of the IC component can be obtained by splitting the IC emission into components corresponding to the three ISRF components (starlight, infrared, and the cosmic microwave background), and comparing it with the model when the three components are joined together, or when the IC emission is split into Galactocentric rings [11]. The effect of these changes can be quite significant, as shown in Figure 3 on the right.

\section{Modeling of resolved PS near the GC}

Uncertainties in the diffuse model can significantly affect detection and characterization of point sources near the GC, which in their turn can influence the properties of the GC excess, especially at energies below $1 \mathrm{GeV}$, where the contribution of point sources is relatively large. In ref. 
[10] several diffuse models were tested with a list of PS derived for each of the models. Different diffuse models and PS search algorithms were also considered in [11]. One can see that the influence of the PS modeling in conjunction with the changes in diffuse emission models (shown in Figure 4) is larger than the changes due to diffuse model alone (Figure 2).
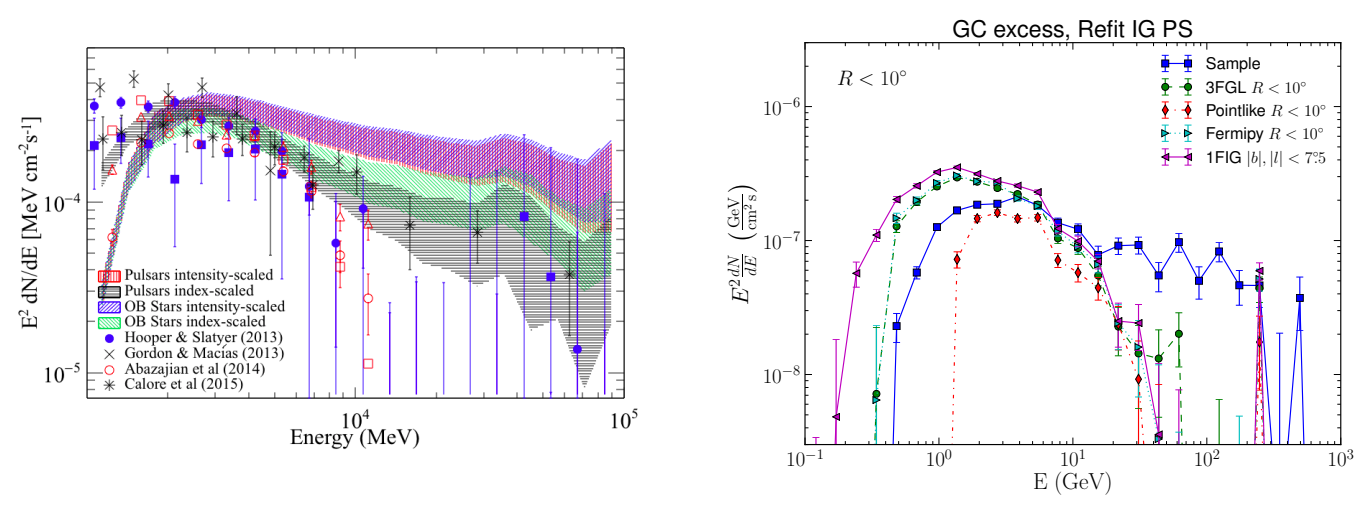

Figure 4: Effect of diffuse emission modeling and PS characterization on the GC excess in [10] (left) and [11] (right).

\section{Sources of CR near the GC}

There are very dense molecular clouds around the GC, the central molecular zone (CMZ). These molecular clouds are known to host star formation and, as a result, can be a source of CR via supernovae explosions.

Several analysis have found a strong influence of the sources of CR near the GC on the GC excess $[20,21,22,11]$. However, none of the models was able to explain simultaneously the spectrum and morphology of the excess. Some examples of the effect on the GC excess spectrum are shown in Figure 5. In particular, in the plot on the right, the CMZ source mostly affects the GC excess at energies below about $5 \mathrm{GeV}$, while a source of $\mathrm{CR}$ electrons from the Galactic bulge (e.g., coming from millisecond pulsars in the bulge [23]) is degenerate with the GC excess at energies above about $5 \mathrm{GeV}$.
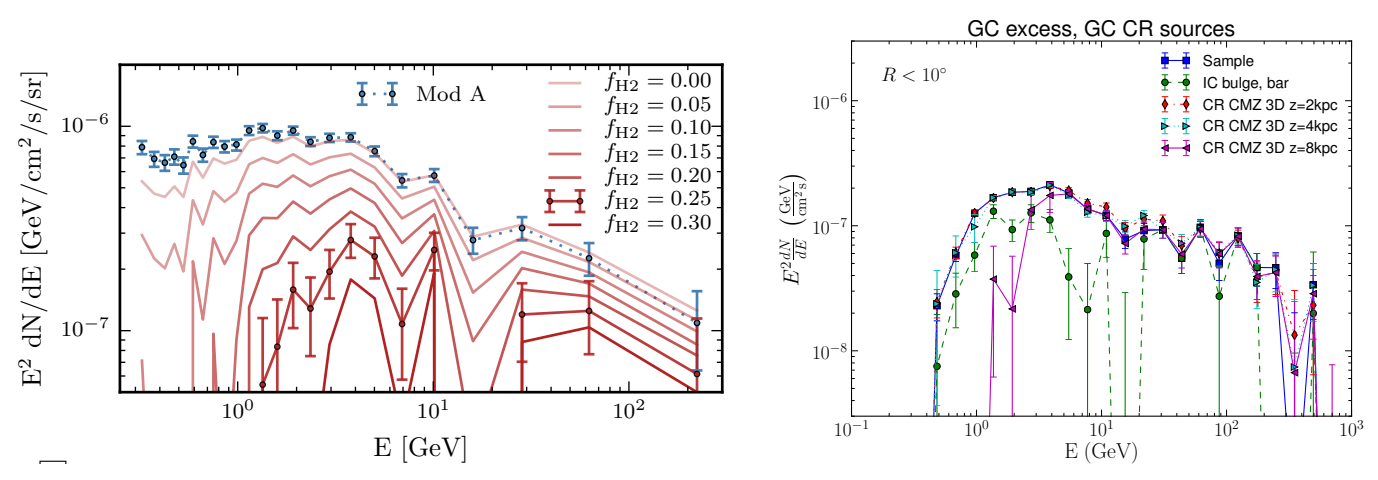

Figure 5: The flux attributed to the GC excess can be significantly reduced after adding new sources of CR near the GC as discussed in [20] (left) and [11] (right). 


\section{Fermi bubbles}

One of the main difficulties in modeling the contribution of the FB near the GC is that neither their spectrum no the morphology is known in that region. Assuming that the spectrum of the bubbles is the same at low latitudes and at high latitudes (at least in the energy range from $1 \mathrm{GeV}$ to $10 \mathrm{GeV}$ ) an all-sky template of the bubbles can be derived [11]. The corresponding all-sky template of the bubbles and the effect on the GC excess are shown in Figure 6. The bubbles template is consistent with the morphology of the bubbles derived earlier [24].
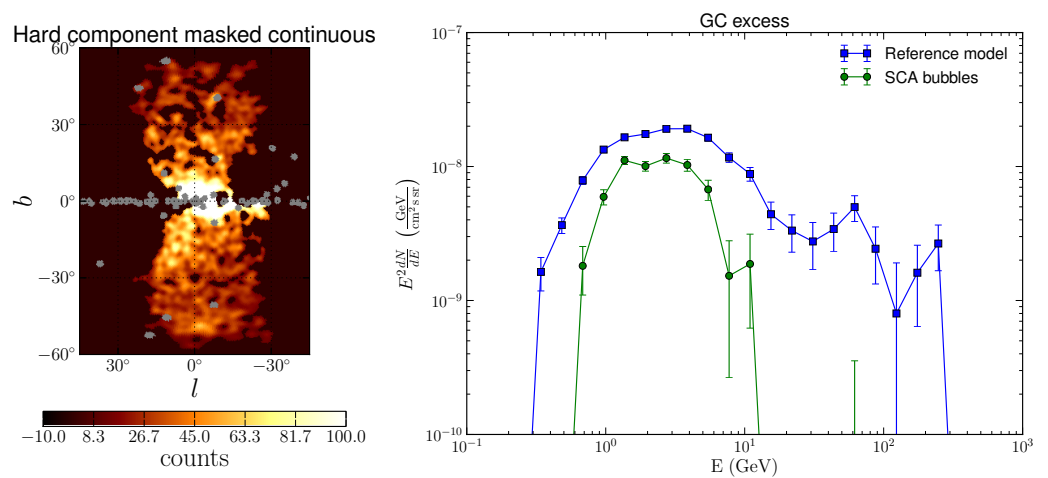

Figure 6: All-sky bubbles template (left) and the effect on the GC excess spectrum (right) [11].

One can notice that the FB morphology is not symmetric with respect to the GC. They also absorb all of the GC excess above $10 \mathrm{GeV}$, while significantly reducing the flux below $10 \mathrm{GeV}$. An interpretation can be that the excess emission near the GC consists of two parts, the FB emission, which is shifted to negative longitudes and has a spectrum similar to the spectrum of the FB at high latitudes, and the remaining excess emission, which has a morphology consistent with spherical symmetry around the GC [11] and a spectrum that has a cutoff around a few GeV. This interpretation is further discussed in the next section.

\section{GC excess morphology}

The morphology of the excess is generally consistent with spherical symmetry around the GC, e.g., Figure 7. One can notice, however, that the spectrum of the the GC excess in region VII (to the left of the GC) is consistent with zero above $10 \mathrm{GeV}$, while it is significantly above zero in other regions, e.g., I, II, III, IV, V, VI, VIII. This observation is consistent with the hypothesis stated in the previous section that the GC excess emission above $10 \mathrm{GeV}$ can be explained by the FB displaced slightly to the right from the GC, while the emission below $10 \mathrm{GeV}$ is a combination of the FB and a spherical GC excess with a cutoff in spectrum around a few GeV. The possible bubble-like structure of the excess was also noticed in refs. [24, 25, 26]. However, it is difficult to distinguish a purely hourglass shape from a spherical shape of the excess due to large uncertainties in gamma-ray diffuse emission along the Galactic plane [11]. Thus the hypothesis that the excess consists of two components: one with an hourglass shape and a spherical one is plausible. 

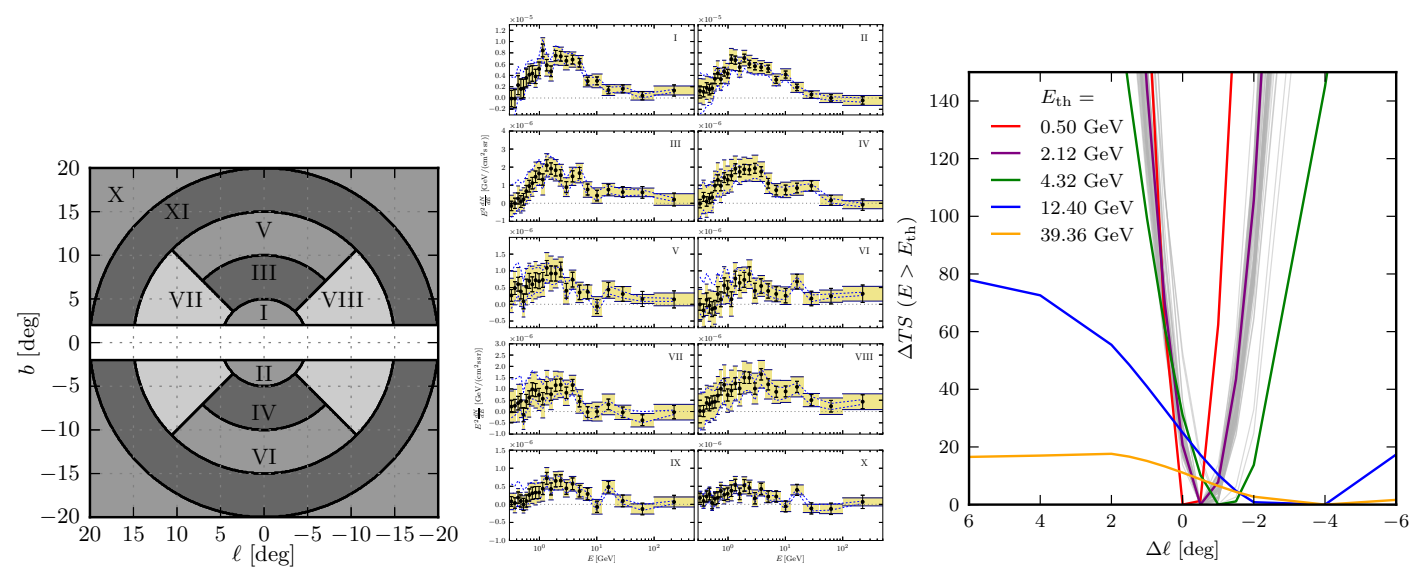

Figure 7: Left: different regions around GC, middle: GC excess spectra in these regions, right: best fit positions of the center of a DM annihilation cusp profile [8].

A further support for the hypothesis of the two component contribution to the excess can be indirectly found by looking at the best-fit position of the GC excess center. At energies below $10 \mathrm{GeV}$ the excess is slightly $\left(\lesssim 1^{\circ}\right)$ shifted to the right from the GC: here there are two components contributing to the excess; while at energies above $10 \mathrm{GeV}$ the shift is more significant $\left(\gtrsim 2^{\circ}\right)$ : this is where only the FB component contributes.

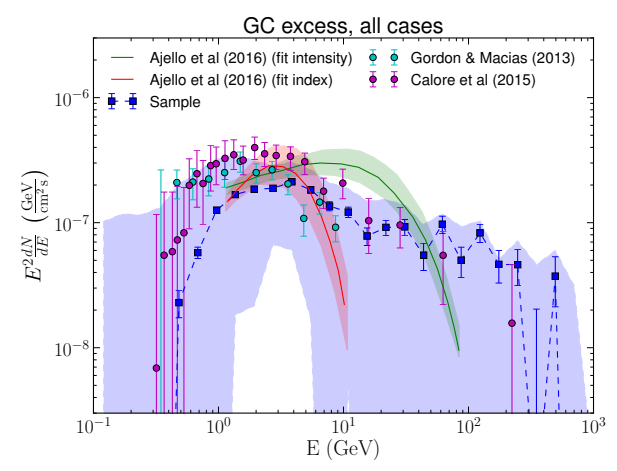

Figure 8: GC excess spectra systematic band [11] compared to GC excess spectra in $[6,8,10]$.

\section{Conclusions}

GC excess has been found around a few $\mathrm{GeV}$ in all models of diffuse emission considered by several groups, e.g., Figure 8. The spectrum of the excess has significantly uncertainties at low and at high energies. The main factors affecting the excess at energies below $1 \mathrm{GeV}$ are characterization of resolved PS, modeling of diffuse emission components ( $\pi^{0}$ and IC), additional CR sources near the GC. At high energies, an important contribution can also come from additional sources of CR near the GC and the Fermi bubbles.

Correlation between the GC excess and a model of the Fermi bubbles at energies above $10 \mathrm{GeV}$ suggests an interpretation that the GC excess consists of two components: a spherical component with a spectrum that has a cutoff at a few $\mathrm{GeV}$ and a bi-lobed component with a spectrum similar to the spectrum of the bubbles at high latitudes. This hypothesis is also supported by the studies of the GC excess morphology, such as the best fit position of the center of a DM annihilation cusp profile, which is close to $\mathrm{GC}$ at energies below $10 \mathrm{GeV}$, but moves to negative longitudes for energies above $10 \mathrm{GeV}$. In the future, this hypothesis can be tested if a better model of the Fermi bubbles becomes available, e.g., by observations with Cherenkov telescopes at energies above $100 \mathrm{GeV}$.

At the moment, several explanations of the GC exist, including DM annihilation $[1,6,7,8]$ and a contribution of unresolved point sources [27, 28, 29], such as millisecond pulsars (MSPs) 
[30, 31]. Future searches of MSPs in the bulge with radio telescopes may confirm or rule out the MSP interpretation of the GC excess [32].

Acknowledgements. The Fermi-LAT Collaboration acknowledges support from NASA and DOE (United States), CEA/Irfu, IN2P3/CNRS, and CNES (France), ASI, INFN, and INAF (Italy), MEXT, KEK, and JAXA (Japan), and the K.A. Wallenberg Foundation, the Swedish Research Council, and the National Space Board (Sweden).

\section{References}

[1] L. Goodenough and D. Hooper, Possible Evidence For Dark Matter Annihilation In The Inner Milky Way From The Fermi Gamma Ray Space Telescope, ArXiv:0910.2998 (2009) , [0910 .2998].

[2] FERMI-LAT collaboration, V. Vitale and A. Morselli, Indirect Search for Dark Matter from the center of the Milky Way with the Fermi-Large Area Telescope, in Fermi gamma-ray space telescope. Proceedings, 2nd Fermi Symposium, Washington, USA, November 2-5, 2009, 2009, 0912.3828.

[3] D. Hooper and L. Goodenough, Dark Matter Annihilation in The Galactic Center As Seen by the Fermi Gamma Ray Space Telescope, Phys. Lett. B697 (2011) 412-428, [1010 . 2752].

[4] K. N. Abazajian and M. Kaplinghat, Detection of a Gamma-Ray Source in the Galactic Center Consistent with Extended Emission from Dark Matter Annihilation and Concentrated Astrophysical Emission, Phys. Rev. D86 (2012) 083511, [1207.6047].

[5] D. Hooper and T. R. Slatyer, Two Emission Mechanisms in the Fermi Bubbles: A Possible Signal of Annihilating Dark Matter, Phys. Dark Univ. 2 (2013) 118-138, [1302 . 6589].

[6] C. Gordon and O. Macias, Dark Matter and Pulsar Model Constraints from Galactic Center Fermi-LAT Gamma Ray Observations, Phys. Rev. D88 (2013) 083521, [1306. 5725].

[7] T. Daylan, D. P. Finkbeiner, D. Hooper, T. Linden, S. K. N. Portillo, N. L. Rodd et al., The characterization of the gamma-ray signal from the central Milky Way: A case for annihilating dark matter, Phys. Dark Univ. 12 (2016) 1-23, [1402.6703].

[8] F. Calore, I. Cholis and C. Weniger, Background Model Systematics for the Fermi GeV Excess, JCAP 1503 (2015) 038, [1 409.0042$].$

[9] B. Zhou, Y.-F. Liang, X. Huang, X. Li, Y.-Z. Fan, L. Feng et al., GeV excess in the Milky Way: The role of diffuse galactic gamma-ray emission templates, Phys. Rev. D91 (2015) 123010, [1406.6948].

[10] FERMI-LAT collaboration, M. Ajello et al., Fermi-LAT Observations of High-Energy $\gamma$-Ray Emission Toward the Galactic Center, Astrophys. J. 819 (2016) 44, [1511. 02938].

[11] FERMI-LAT collaboration, M. Ackermann et al., The Fermi Galactic Center GeV Excess and Implications for Dark Matter, Astrophys. J. 840 (2017) 43, [1704 . 03910].

[12] M. Su, T. R. Slatyer and D. P. Finkbeiner, Giant Gamma-ray Bubbles from Fermi-LAT: AGN Activity or Bipolar Galactic Wind?, Astrophys. J. 724 (2010) 1044-1082, [1005.5480].

[13] I. Moskalenko and A. Strong, Production and propagation of cosmic ray positrons and electrons, ApJ 493 (1998) 694-707, [astro-ph/9710124].

[14] A. W. Strong, I. V. Moskalenko and V. S. Ptuskin, Cosmic-ray propagation and interactions in the Galaxy, Ann. Rev. Nucl. Part. Sci. 57 (2007) 285-327, [astro-ph/0701517]. 
[15] Fermi-LAT Collaboration collaboration, M. Ackermann, M. Ajello, W. B. Atwood et al., Fermi-LAT Observations of the Diffuse Gamma-Ray Emission: Implications for Cosmic Rays and the Interstellar Medium, ApJ 750 (2012) 3, [1202 . 4039].

[16] F. Yusef-Zadeh et al., Star Formation in the Central 400 pc of the Milky Way: Evidence for a Population of Massive YSOs, Astrophys. J. 702 (2009) 178-225, [0 905.2161$].$

[17] K. Immer, F. Schuller, A. Omont and K. M. Menten, Recent star formation in the inner Galactic Bulge seen by ISOGAL II - The Central Molecular Zone, Astron. Astrophys. 537 (2012) A121, [1111.3295].

[18] S. N. Longmore et al., Variations in the Galactic star formation rate and density thresholds for star formation, Mon. Not. Roy. Astron. Soc. 429 (2013) 987, [1208. 4256].

[19] T. A. Porter, G. Johannesson and I. V. Moskalenko, High-Energy Gamma Rays from the Milky Way: Three-Dimensional Spatial Models for the Cosmic-Ray and Radiation Field Densities in the Interstellar Medium, Astrophys. J. 846 (2017) 67, [1708.00816].

[20] E. Carlson, T. Linden and S. Profumo, Cosmic-Ray Injection from Star-Forming Regions, Phys. Rev. Lett. 117 (2016) 111101, [1510.04698].

[21] D. Gaggero, M. Taoso, A. Urbano, M. Valli and P. Ullio, Towards a realistic astrophysical interpretation of the gamma-ray Galactic center excess, JCAP 1512 (2015) 056, [1507. 06129 ].

[22] I. Cholis, C. Evoli, F. Calore, T. Linden, C. Weniger and D. Hooper, The Galactic Center GeV Excess from a Series of Leptonic Cosmic-Ray Outbursts, JCAP 1512 (2015) 005, [1506.05119].

[23] J. Petrović, P. D. Serpico and G. Zaharijas, Millisecond pulsars and the Galactic Center gamma-ray excess: the importance of luminosity function and secondary emission, JCAP 1502 (2015) 023, [1411.2980].

[24] FERMI-LAT collaboration, F. Acero et al., Development of the Model of Galactic Interstellar Emission for Standard Point-Source Analysis of Fermi Large Area Telescope Data, Astrophys. J. Suppl. 223 (2016) 26, [1602.07246].

[25] R.-z. Yang and F. Aharonian, On the GeV excess in the diffuse $\gamma$-ray emission towards the Galactic centre, Astron. Astrophys. 589 (2016) A117, [1602.06764].

[26] O. Macias, C. Gordon, R. M. Crocker, B. Coleman, D. Paterson, S. Horiuchi et al., Discovery of Gamma-Ray Emission from the X-shaped Bulge of the Milky Way, 1611.06644.

[27] R. Bartels, S. Krishnamurthy and C. Weniger, Strong support for the millisecond pulsar origin of the Galactic center GeV excess, Phys. Rev. Lett. 116 (2016) 051102, [1506. 0510 4].

[28] S. K. Lee, M. Lisanti, B. R. Safdi, T. R. Slatyer and W. Xue, Evidence for Unresolved $\gamma$-Ray Point Sources in the Inner Galaxy, Phys. Rev. Lett. 116 (2016) 051103, [1506.05124].

[29] S. Caron, G. A. Gómez-Vargas, L. Hendriks and R. Ruiz de Austri, Analyzing gamma-rays of the Galactic Center with Deep Learning, 1708.06706.

[30] N. Mirabal, Dark matter vs. Pulsars: Catching the impostor, Mon. Not. Roy. Astron. Soc. 436 (2013) 2461, [1309.3428].

[31] T. D. Brandt and B. Kocsis, Disrupted Globular Clusters Can Explain the Galactic Center Gamma Ray Excess, Astrophys. J. 812 (2015) 15, [1507.05616].

[32] F. Calore, M. Di Mauro, F. Donato, J. W. T. Hessels and C. Weniger, Radio detection prospects for a bulge population of millisecond pulsars as suggested by Fermi LAT observations of the inner Galaxy, Astrophys. J. 827 (2016) 143, [1512.06825]. 